\title{
Desenvolvimento e validação de aplicativo móvel para cálculo de dose pediátrica na unidade de terapia intensiva pediátrica e urgência e emergência
}

\author{
Development and validation of a mobile application for calculating pediatric doses in the \\ pediatric intensive care unit and urgency and emergency \\ Desarrollo y validación de una aplicación móvil para el cálculo de dosis pediátricas en la \\ unidad de cuidados intensivos pediátricos y de urgencia y emergencia
}

Fabiana Nascimento Benedik ${ }^{1}$, Mariseth Carvalho de Andrade ${ }^{1}$, Lêda Lima da Silva ${ }^{1}$, Adilson Mendes de Figueiredo Júnior ${ }^{1 *}$, Anderson Bentes de Lima ${ }^{1}$.

\section{RESUMO}

Objetivo: Desenvolver e validar um aplicativo para cálculo de dose pediátrica na unidade de terapia intensiva pediátrica e urgência e emergência. Métodos: Trata-se de um estudo metodológico, descritivo, aplicado na modalidade de produção tecnológica. Resultados: Dez médicos pediatras utilizaram o aplicativo e posteriormente o avaliaram, segundo os critérios de layout, usabilidade, percepção de utilidade, facilidade de uso e utilidade percebida pela tecnologia, mediante a utilização de um questionário impresso baseado no System Usability Scale - SUS (Escala de Usabilidade de Sistema) e na Technology Acceptance Model - TAM (Modelo de Aceitação de Tecnologia). No geral, o software obteve $88,1 \%$ de aprovação pelos juízes, com $90,0 \%$ de aprovação no quesito usabilidade e facilidade de aprendizagem, e 85,8\% para Utilidade do aplicativo. Conclusão: $\mathrm{O}$ aplicativo móvel CALCULEPED mostrou-se como uma tecnologia útil, prático e bem aceito pelos médicos pediatras, facilitando o cálculo de dose pediátrica nos serviços de urgência, emergência e unidade de terapia intensiva.

Palavras-chave: Aplicativos móveis, Pediatria, Unidades de terapia intensiva pediátrica.

\section{ABSTRACT}

Objective: To develop and validate an application for calculating the pediatric dose in the pediatric intensive care unit and urgency and emergency. Methods: This is a methodological, descriptive study, applied in the mode of technological production. Results: Ten pediatric doctors used the application and subsequently evaluated it, according to the criteria of layout, usability, perceived utility, ease of use and utility perceived by technology, using a printed questionnaire based on the System Usability Scale - SUS (Scale System Usability) and the Technology Acceptance Model - TAM (Technology Acceptance Model). Overall, the software obtained $88.1 \%$ approval by the judges, with $90.0 \%$ approval in terms of usability and ease of learning, and $85.8 \%$ for application utility. Conclusion: The CALCULEPED mobile app proved to be a useful, practical and well-accepted technology by pediatric doctors, facilitating the calculation of pediatric doses in emergency, emergency and intensive care units.

Keywords: Mobile apps, Pediatrics, Pediatric intensive care units.

\section{RESUMEN}

Objetivo: Desarrollar y validar una aplicación para el cálculo de la dosis pediátrica en la unidad de cuidados intensivos pediátricos y urgencia y emergencia. Métodos: Se trata de un estudio metodológico descriptivo, aplicado en el modo de producción tecnológica. Resultados: Diez pediatras utilizaron la aplicación y posteriormente la evaluaron, según los criterios de maquetación, usabilidad, utilidad percibida, facilidad de uso y utilidad percibida por la tecnología, utilizando un cuestionario impreso basado en la Escala de Usabilidad del Sistema - SUS (Scale Usabilidad del Sistema) y el Modelo de Aceptación de Tecnología TAM (Modelo de Aceptación de Tecnología). En general, el software obtuvo un 88,1\% de aprobación por

${ }^{1}$ Universidade do Estado do Pará (UEPA), Belém - PA. *E-mail: adilsonmdfj@hotmail.com 
parte de los jueces, con un 90,0\% de aprobación en términos de usabilidad y facilidad de aprendizaje y un 85,8\% en la utilidad de la aplicación. Conclusión: La aplicación móvil CALCULEPED demostró ser una tecnología útil, práctica y bien aceptada por los pediatras, facilitando el cálculo de dosis pediátricas en las unidades de urgencias, urgencias y cuidados intensivos.

Palabras clave: Aplicaciones móviles, Pediatría, Unidades de cuidados intensivos pediátricos.

\section{INTRODUÇÃO}

A condição clínica do paciente pediátrico habitualmente já é um fator que pode aumentar os riscos de evento adverso, se complica ainda mais pela escassez de estudos clínicos sobre o uso de medicamentos nessa faixa etária, pelas formas farmacêuticas disponíveis em dosagens e concentrações adequadas para a administração, e pela necessidade de cálculo de doses individualizadas conforme idade, peso e área de superfície corpórea, dessa forma, observa-se que é necessária atenção e destreza quando o assunto é medicação pediátrica, principalmente, pelos riscos envolvidos que podem variar desde alterações dermatológicas até o risco eminente de morte (IPSM, 2015).

$O$ atendimento inicial prestado em urgências e emergências e unidades de terapia intensiva pediátrica são caracterizados por serem ambientes desafiadores com terapias de múltiplas drogas, doenças complexas, alta acuidade de atendimento e possíveis fatores latentes, como estresse, ruído, pressões de tempo e pacientes desconhecidos (HEINZEL O, et al., 2015; WILIAM W, et al., 2017).

Em diversos estudos, foi comprovado que as crianças usam drogas de forma inadequada, principalmente por se tratarem da automedicação. O grande consumo de drogas na infância pode ser atribuído ao padrão da doença e às manifestações clínicas dessa faixa etária. Além dos padrões condicionais, o estoque de medicamentos em casa pode ser outro fator que desencadeia 0 tratamento médico e a automedicação (MANIERO HK, et al., 2018).

A automedicação pediátrica, por exemplo, consiste na administração de medicamentos para a criança ou adolescente, não há observação médica prévia. Essa prática pode ser devido à compra de medicamentos sem receita por familiares ou membros sociais que compartilham drogas e uso repetido de medicamentos previamente tratados, este tópico é particularmente importante porque nas últimas décadas, embora faltem evidências da eficácia de certos medicamentos no ambiente de mercado em que são comercializados, em muitos países desenvolvidos a automedicação para crianças e adolescentes aumentou (BELO N, et al., 2017).

Os medicamentos desempenham um papel fundamental na restauração e manutenção da saúde e devem ser seguros, eficazes, de alta qualidade e de baixo custo para pacientes e comunidades. $O$ uso indevido traz riscos, principalmente para crianças, pois está relacionado à absorção, metabolismo, excreção e até mesmo o efeito das drogas nos receptores desses pacientes é diferente da dos adultos, o que torna esse tipo de população particularmente mais vulnerável ao uso de drogas (MANIERO HK, et al., 2018).

A maioria dos medicamentos administrados por via intravenosa para as crianças são fornecidas em frascos originalmente preparados para a população adulta. Isso leva à necessidade de cálculo e preparação da dose do medicamento com base no peso para cada criança, o que varia amplamente entre as faixas etárias. Aliado a isso, crianças apresentam características peculiares que alteram sua capacidade de absorção, metabolização e excreção de drogas, além de não terem a capacidade de relatar possíveis efeitos (IPSM, 2015).

A segurança do paciente é considerada um domínio central da qualidade de cuidados e pode ser definido como a redução de eventos adversos associado à saúde para um mínimo aceitável. O avanço da tecnologia permitiu a criação de novos sistemas para auxiliar no processo de medicação, e por causa da disponibilidade de dispositivos móveis e a possibilidade de combinar banco de informações e suporte para auxiliar cálculos, aplicativos móveis têm um alto potencial para melhorar a segurança dos pacientes em casos de emergência (KAHN S e ABRAMSON EL, 2019).

Pela característica de agilidade nas tomadas de decisão, esses setores necessitam de uma maior atenção, o que torna um desafio para o médico pediatra, porém mais ainda desafiador para clínicos gerais, 
os quais ou não estão familiarizados com os cálculos de doses individualizadas para a população pediátrica ou são inexperientes quanto aos diversos cálculos que devem ser realizados para a obtenção da dose. Diante disto, a taxa de erros de medicação aumenta mais ainda em ambientes de cuidados intensivos, os quais exige a execução de cálculos complexos e é uma fonte de erros (HEINZEL O, et al., 2015).

Erros de medicação, além de proporcionarem altos custos, também podem ocasionar transtornos, como mudança no resultado terapêutico dos pacientes e aumento de sua morbimortalidade, e problemas psicológicos aos profissionais envolvidos. Desta forma, torna-se imprescindível que se invista em tecnologias, auxiliando no aprimoramento do cuidado e à promoção da cultura da segurança ao paciente (KAHN S e ABRAMSON EL, 2019).

Um ponto crucial em pediatria é a ocorrência frequente de erros em cálculos que utilizam valores com casas decimais ou conversões de unidades de medida, como por exemplo, de Miligrama (mg) para Kilograma $(\mathrm{kg})$, Miligrama $(\mathrm{mg})$ em Mililitro $(\mathrm{ml})$. Esses cálculos matemáticos estão diretamente ligados ao peso e às concentrações já estipuladas pela indústria farmacêutica, que necessitam na maior parte das vezes de mais de um cálculo para se obter o valor adequado para a criança. Portanto, a importância de ter uma ferramenta que auxilie na redução de probabilidade de erros, que aperfeiçoe o atendimento ao paciente pediátrico, torna-se fundamental para a promoção da segurança do paciente, visto que erro de medicação é definido como um evento evitável (HEINZEL O, et al., 2015; WEGNER W, et al., 2017).

Um aplicativo móvel chamado PedAMINES, desenvolvido em hospitais da Universidade de Genebra na Suíça, para a utilização no cálculo de drogas utilizadas na ressuscitação cardiopulmonar pediátrica em ambientes de emergência criados fora do hospital, apresentou validação satisfatória, demonstrando eficiência na redução de erros nos cálculos das doses e menor tempo na administração do medicamento, apresentando $60 \%$ de maior probabilidade aos erros, ao grupo de médicos que não fizeram uso do aplicativo. Com base no estudo citado, compreende-se a importância de estudos em ferramentas tecnológicas que promovam segurança e melhoria dentro da saúde e com isso, o desenvolvimento de um dispositivo móvel, suporte no atendimento ao paciente pediátrico, conferindo agilidade e segurança (SIEBERT JN, et al., 2019). O objetivo do estudo foi desenvolver e validar um aplicativo de celular para cálculo de dose pediátrica na urgência e emergência e unidade de terapia intensiva pediátrica.

\section{MÉTODOS}

Foi desenvolvido estudo de avaliação de tecnologia, prospectivo, transversal, indutivo e observacional. A pesquisa foi iniciada após aceite da instituição preponente, pelo Comitê de Ética em Pesquisa, através da Plataforma Brasil (CAAE:92451418.8.0000.5171) e autorizada pelos participantes por meio da leitura e assinatura do Termo de Consentimento Livre e Esclarecido (TCLE). O desenvolvimento e validação do aplicativo ocorreram em quatro fases.

A primeira fase do desenvolvimento do aplicativo consistiu na escolha do software, no seu desenvolvimento de criação e no levantamento bibliográfico acerca do tema. Baseado nas referências bibliográficas Blackbook - Pediatria; NeoFax: guia de medicações usadas em recém-nascidos e no bulário eletrônico registrado na Agência Nacional de Vigilância Sanitária (ANVISA), foi realizada uma busca por medicamentos utilizados para tratamentos de urgência e emergência, tais como, medicamentos utilizados no choque e paradas cardiorrespiratórias, hipotensão sintomática, antagonistas e antídotos, tratamento dos desequilíbrios hidroeletrolíticos, anti-hemorrágicos; anestésicos locais e sistêmicos, inalatórios e bloqueadores neuromusculares.

A segunda fase baseou-se no desenvolvimento do aplicativo através de um contrato de prestação de serviços com uma empresa desenvolvedora de softwares. Foi apresentado todo o material coletado, resultado da busca bibliográfica, e iniciado através de reuniões realizadas mensalmente, o desenvolvimento de cada etapa do aplicativo, como o visual, layout e suas funcionalidades. Após o desenvolvimento, todos os aspectos do aplicativo foram analisados pela empresa desenvolvedora, conjuntamente pelos autores, até a obtenção do resultado final. 
A terceira fase fundamentou-se na avaliação do aplicativo, por meio de 10 juízes eleitos através de critério de inclusão, ou seja, serem todos médicos pediatras atuantes na unidade de terapia intensiva pediátrica, com no mínimo cinco anos de formação acadêmica, e como critério de exclusão, profissionais médicos não pediatras, com menos de cinco anos de formação acadêmica, e não atuantes nas unidades de terapia intensiva pediátrica.

Os juízes avaliaram o aplicativo segundo os critérios de usabilidade, percepção de utilidade e facilidade de aprendizagem. Inicialmente, foram convidados a participar do estudo de forma separadamente, dentro do ambiente hospitalar. Após a leitura e assinatura do termo de consentimento livre e esclarecido, foram apresentadas as orientações e demonstrado as funcionalidades e o manuseio do software, concedendo tempo necessário para que o juiz avaliasse o aplicativo.

A quarta fase constituiu-se da entrega de um questionário impresso para preenchimento da avaliação realizada. O aplicativo foi dividido em 5 abas: "Calculadora Geral", "Lista dos Medicamentos", "Rosa dos Ventos", "Superfície Corpórea" e "Sobre o APP", com a interface inicial do aplicativo seguido das funções de acordo com a Figura 1.

Dentro das questões abertas, os médicos pediatras foram convidados à responder se o aplicativo poderia ser útil na prática clínica no dia a dia, e quais pontos positivos, negativos ou sugestões poderiam ser colocadas. Não foram levantados pontos negativos e a maioria respondeu apontando sobre o aplicativo ser prático e seguro, fácil acesso, rápido para checagem na emergência, útil para outras especialidades médicas, redução de tempo no atendimento e que possui medicações muito utilizadas na rotina da emergência pediátrica e que necessitam de cálculos rápidos.

Os juízes admitiram a aplicabilidade clínica que a tecnologia dispõe e demonstraram interesse para utilizá-lo e repassarem para outros profissionais médicos sobre o aplicativo móvel.

Figura 1 - Interface inicial e grupo de funções do aplicativo CALCULEPED.

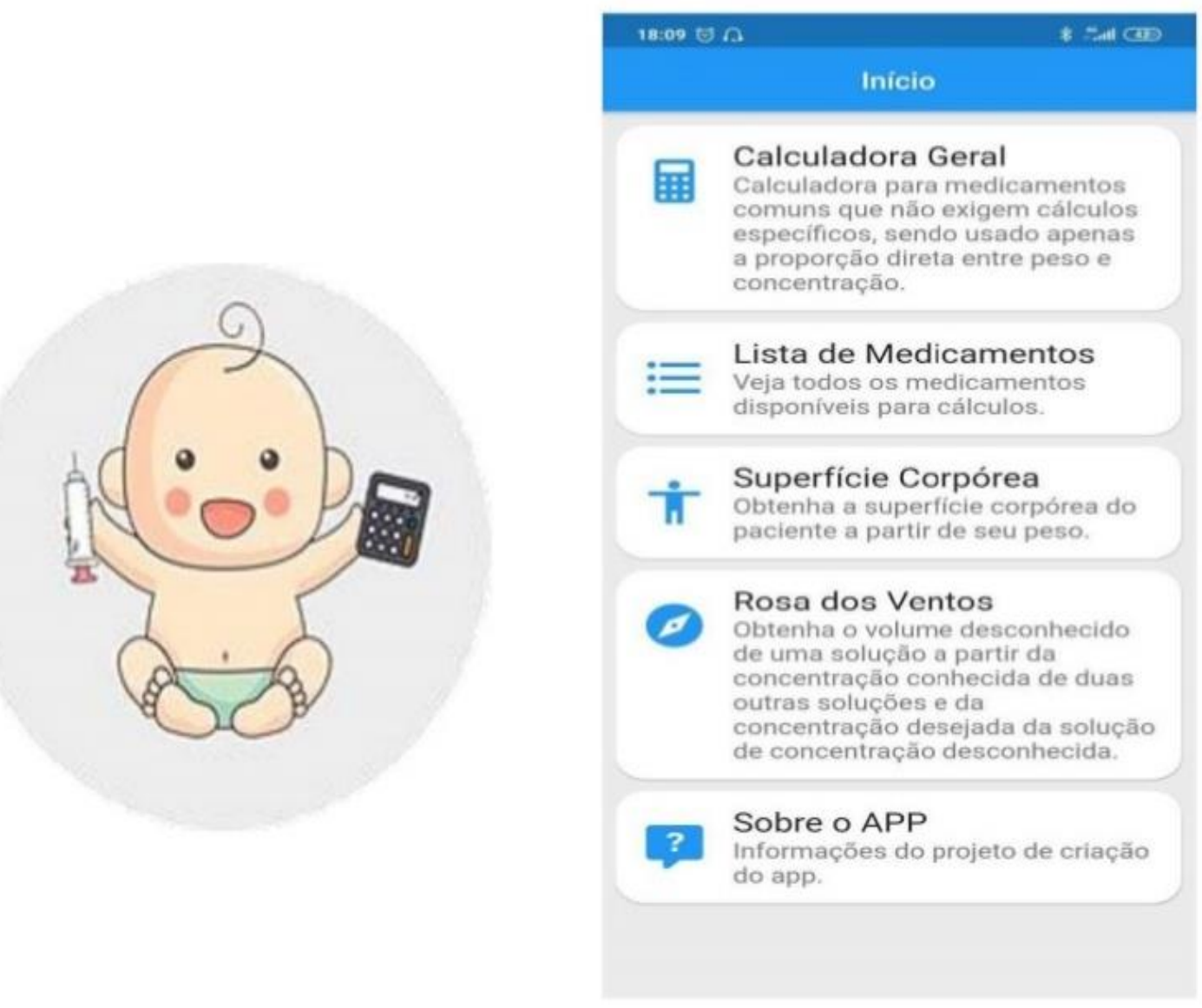

Fonte: Benedik FN, et al., 2020. 


\section{RESULTADOS}

Inicialmente foi caraterizado o perfil dos avaliadores, onde investigou-se o sexo, faixa etária, tempo de formação, formação em residência, título de especialista e qual especialidade (Tabela 1) e em seguida a primeira parte da validação averiguou a usabilidade e facilidade de aprendizagem, apontando os aspectos positivos e negativos do aplicativo. Quanto aos pontos positivos, os médicos pediatras avaliaram com percentual de $92,0 \%$ no critério de "Gostaria de usar este sistema frequentemente", 92,0\% no critério "sistema fácil de ser utilizado", $94 \%$ para a integralidade das funções no aplicativo. Na parte que tange se a maioria dos médicos pediatras aprenderia a utilizar o aplicativo rapidamente, o percentual foi de $94 \%$ e por fim, para o quesito muita confiança nas operações matemáticas e doses calculadas neste aplicativo obtiveram um percentual de $84 \%$ de acordo com a Tabela 2.

Tabela 1 - Perfil dos avaliadores do aplicativo CALCULEPED.

Perfil dos avaliadores

\section{Sexo}

Feminino

Masculino

Faixa etária (anos)

$$
<40
$$

40 a 49

$>=50$
Frequência

$$
\%(n=10)
$$

\section{Tempo de formação (anos)}

$$
<10
$$

10 a 19

20 a 29

$>=30$

9

1
$90,0 \%$

$10,0 \%$

$\begin{array}{ll}9 & 90,0 \% \\ 1 & 10,0 \%\end{array}$

3

$30,0 \%$

3

$30,0 \%$

4

$40,0 \%$

$30,0 \%$
$30,0 \%$
$40,0 \%$

\section{Formação em Residência}

Pediatria

UTI Pediátrica

Título de Especialista

\begin{tabular}{lll}
\hline Sim & 8 & $80,0 \%$ \\
Não & 2 & $20,0 \%$ \\
\hline Qual especialidade & & \\
\hline Pediatria & 5 & $50,0 \%$ \\
Terapia intensiva Pediátrica & 4 & $40,0 \%$ \\
Neonatologia & 1 & $10,0 \%$
\end{tabular}

Fonte: Benedik FN, et al., 2020. 
Tabela 2 - Avaliação da usabilidade e facilidade de aprendizagem do aplicativo CALCULEPED.

$\begin{array}{lcc} & \text { Pontuação } & \% \\ \text { Usabilidade e facilidade de aprendizagem } & \text { Recebida } & \text { Alcançado }\end{array}$

Fatores positivos:

1.1 - Gostaria de usar este sistema frequentemente. $46 \quad 92,0 \%$

1.3 - O Aplicativo possui um sistema fácil de ser utilizado.

$46 \quad 92,0 \%$

1.5 - As diversas funções no sistema do aplicativo foram bem integradas.

$47 \quad 94,0 \%$

1.7 - Houve total semelhança neste aplicativo em relação às doses que costumo prescrever.

$4590,0 \%$

1.8 - A maioria dos pediatras aprenderia a usar esse Aplicativo rapidamente.

$47 \quad 94,0 \%$

1.10 - Muita confiança nas operações matemáticas e doses calculadas deste aplicativo.

$42 \quad 84,0 \%$

Pontuação fatores positivos:

273

$91,0 \%$

Fatores negativos:

1.2 - O Aplicativo possui um sistema desnecessariamente complexo.

$28,0 \%$

1.4 - É preciso apoio de um suporte técnico para ser possível usar este Aplicativo.

1.6 - Muitas inconsistências neste aplicativo em relação às doses que costumo prescrever.

$14 \quad 28,0 \%$

1.9 - O aplicativo é muito pesado para uso.

15

$30,0 \%$

Pontuação fatores negativos:

60

$30,0 \%$

\section{GERAL}

$0 \%$ a $50 \%$ Não Validado

$51 \%$ a $75 \%$ Validado com resalvas

$76 \%$ a $100 \%$ Validado

\begin{tabular}{ccc}
\hline Avaliação Geral Parte 1 & 413 & $82,6 \%$ \\
\hline
\end{tabular}

Fonte: Benedik FN, et al., 2020.

A segunda etapa da validação consistiu em analisar sobre a utilidade do aplicativo. Quanto aos pontos positivos, no item "facilidade para o cálculo de doses pediátricas precisas", o resultado foi de $92 \%$, no tópico "o aplicativo calculou mais precisamente as doses de medicamentos e eletrólitos", a porcentagem foi de $90 \%$. No ponto "calcula mais rapidamente as doses de medicamentos e eletrólitos", obteve-se $94 \%$.

Quanto se a tecnologia seria útil dentro da urgência e emergência, melhorando a assistência ao paciente pediátrico, o resultado foi de $92 \%$. A respeito se a tecnologia apresenta informações em consonância com as melhoras evidências científicas, foi atingido um percentual de $90 \%$, e por fim, quanto à aceitação e utilização entre os médicos pediatras, o resultado alcançado foi de $84 \%$, de acordo com a Tabela 3. 
Tabela 3 - Avaliação da utilidade do aplicativo CALCULEPED.

Sobre a utilidade do aplicativo

Pontuação
Recebida
$(0-50)$

\section{Fatores positivos:}

Facilitou o cálculo de doses pediátricas precisas.

Informações atualizadas e em consonância com evidências científicas.

Será bem aceito e utilizado entre os pediatras.

Pontuação fatores positivos:
46

45

47

46

45

$92,0 \%$

$90,0 \%$

$94,0 \%$

$92,0 \%$

$90,0 \%$

43

$86,0 \%$

272

$90,7 \%$

Fatores negativos:

Difícil encontrar doses utilizadas na prática clínica.

16

13

29

343
$32,0 \%$

$26,0 \%$

$29,0 \%$

$85,8 \%$

Fonte: Benedik FN, et al., 2020.

Assim, na avaliação geral, o aplicativo alcançou 90,0\% no item de usabilidade e facilidade de aprendizagem e $85,8 \%$ no item utilidade do aplicativo, conquistando no final $88,1 \%$, o que the confere a validação pelos juízes de acordo com a Tabela 4.

Tabela 4 - Critérios para validação do aplicativo CALCULEPED.

\section{Critérios para validação do aplicativo}

1. Usabilidade e Facilidade de aprendizagem

2. Utilidade do aplicativo

\section{Pontuação Recebida} (0-500)

450

343

793
\% Aprovação

$90,0 \%$

$85,8 \%$

$88,1 \%$

Geral

Fonte: Benedik FN, et al., 2020.

A coleta de dados se deu em valores absolutos e percentuais, sendo utilizado o coeficiente alfa de Conbrach para medir a confiabilidade do questionário, e atingindo um valor de 0,83 , configurando boa consistência, inferindo assim, na confiabilidade admissível da validação.

Os juízes admitiram a aplicabilidade clínica que a tecnologia dispõe e demonstraram interesse para utilizá-lo e repassarem para outros profissionais médicos sobre o aplicativo móvel. 


\section{DISCUSSÃO}

As características do atendimento na emergência pediátrica envolvem diversos fatores, como: fatores biológicos e psicológicos, características próprias de populações específicas, vulnerabilidade aos agravos por doenças epidêmicas na infância e agravos entendidos como emergências, como traumas e envenenamentos, além do cálculo de medicamentos e tomada de decisão. Em uma emergência pediátrica, podem ocorrer graves situações de risco de vida e, dessa forma, percebe-se que o tempo é determinante para o sucesso do tratamento (PIRES MCAC, et al., 2017; SERAFIM MPS, et al., 2019).

Em pacientes pediátricos, devido à especificidade e complexidade do grupo etário, eles são mais propensos a serem afetados por defeitos do sistema de administração de medicamentos. Esses grupos etários são vulneráveis ao peso corporal, superfície corporal, mecanismos farmacocinéticos e farmacodinâmicos e absorção, os efeitos das alterações no metabolismo e na excreção do medicamento, assim são necessárias ferramentas capazes de otimizar o cuidado medicamentoso ao público em questão (MACEDO GPO, et al., 2015; VOLPATTO BM, et al., 2017).

Assim, torna-se evidente que é necessário aprimorar a forma de avaliar as variáveis presentes no paciente pediátrico e promover com mais segurança a administração de medicamentos a esse público, visto que historicamente ainda são presentes os erros de cálculo, assim como os erros de vias de administração.

A aplicação de medicamentos requer conhecimento científico e habilidade técnica e, com a cooperação de órgãos oficiais, órgãos reguladores e instituições de saúde do sistema de saúde brasileiro, destaca-se a importância de diretrizes ou recomendações para melhorar a segurança do paciente. Os serviços de saúde públicos ou privados, e com a participação ativa dos profissionais de saúde, promovem o desenvolvimento de estratégias para minimizar esses erros, entre os quais cita-se a capacitação de profissionais envolvidos e a criação de ferramentas capazes de auxiliar na destreza e velocidade da administração de medicamentos (ROCHA CM, et al., 2018).

Ferramentas que podem aprimorar a prática médica como pesquisas, ferramentas digitais e aplicativos podem ser muito úteis. Esses recursos podem otimizar o tempo do profissional - ideal para rotinas complicadas - e facilitar as investigações. Além disso, é uma forma de se manter atualizado com as novidades de procedimentos e informações. Em geral, os benefícios que o software traz para a atuação médica e de outros profissionais são diversos: fornecem um início mais rápido do tratamento, melhoram os serviços médicos, facilitam o monitoramento abrangente, auxiliam na tomada de decisões e reduzem a mortalidade e as internações hospitalares (PEREIRA VSR, et al., 2017).

As ferramentas de informática na área da saúde estão em constante expansão e cada vez mais profissionais e organizações as utilizam. Essa tecnologia permite ao profissional obter maior precisão e agilidade no trabalho. Dentre as ferramentas de computação, a computação móvel se destaca e pode ser aplicada em diferentes áreas da área médica. Nessas aplicações, pode-se destacar o suporte à tomada de decisão, monitoramento remoto, diagnóstico e otimização da segurança do paciente. O aumento do número de dispositivos móveis tornou a informática uma realidade na vida das pessoas nas áreas pessoal, educacional e profissional (SILVA RH, et al., 2020; MATOS JC, et al., 2018).

$\mathrm{O}$ avanço da tecnologia e a sua utilização crescente na saúde tem sido fundamental para a prevenção e diminuição da probabilidade de erros associados ao cálculo de dose pediátrica, colocando a segurança do paciente como domínio central na qualidade de cuidados. Design fácil, prático e que se encaixam no fluxo do atendimento devem ser cada vez mais incentivados para criação de sistemas para a prática clínica, uma vez que esses estudos são escassos e necessários (KAHN S e ABRAMSON EL, 2019; BARBOSA AS, 2019).

Na situação atual, as tecnologias de informação e comunicação (TIC) voltadas para o setor saúde possuem uma variedade de ferramentas que suportam a estrutura e organização de dados e informações para que possam ser armazenados, processados, acessados e compartilhados em tempo real e/ou remotamente. Essas tecnologias são consideradas um recurso global, que pode conectar vários computadores para criar uma rede de informações e pode cooperar com o desenvolvimento e o progresso do setor de saúde (AMORIM DNP, et al., 2018; EMYINUMARU F, et al., 2019). 
Os aplicativos devem ser baseados em evidências científicas, e é importante considerar e valorizar as percepções do usuário diante do uso da tecnologia, tanto voltadas para aceitação ou rejeição da mesma, assim como, a avaliação voltada para os recursos técnicos disponibilizados. Portanto, a validação por meio de questionários estruturados para avaliar a opinião dos juízes é necessária para abranger o ponto de vista dos usuários (PEREIRA VSR, et al., 2017).

De acordo com os estudos de Macedo GPO, et al. (2015), ferramentas que possam aumentar a precisão da administração de medicamentos são necessárias para facilitar o lidar de pacientes pediátricos, e com a utilização de aplicativos móveis a prescrição medicamentosa otimizou em aproximadamente $70 \%$ esse processo. Diferente dos aplicativos existentes para cálculos de dose de medicamentos, o CALCULEPED buscou priorizar $\mathrm{o}$ atendimento pediátrico, disponibilizando apenas drogas utilizadas em urgência e emergência e unidade de terapia intensiva, possibilitando o uso de três calculadoras, sendo uma utilizada para cálculos de dose de acordo com o peso, concentração do medicamento e a patologia selecionada, outra para cálculo de superfície corpórea e por fim, a calculadora "Rosa dos Ventos", utilizada para calcular uma concentração de uma substância desconhecida a partir de outras duas substâncias com concentrações conhecidas.

Os aplicativos voltados para a saúde e o atendimento pediátrico são recursos importantes, pois detectam precocemente os problemas de saúde e promovem a segurança do paciente. Portanto, esses aplicativos podem ser usados não apenas como ferramentas de medição e diagnóstico para os profissionais de saúde, mas também como ferramentas de aconselhamento e esclarecimento para os familiares, de modo que possam aprimorar a capacidade de interpretação no cuidado à saúde (AMORIM DNP, et al., 2018; EMYINUMARU F, et al., 2019).

De acordo com os estudos de Souza ASC, et al. (2018), que avaliou as publicações que envolviam a administração de medicações em urgências pediátricas entre os anos de 2011 a 2017, observou-se que fatores como avaliação de peso, restrições de tempo e carga de trabalho interferem diretamente na precisão do cálculo medicamentoso, o mesmo estudo afirma também que a existência de aplicativos pode resultar em uma forma eficiente de mudança e aperfeiçoamento da administração de medicamentos.

Tais dados corroboram os estudos de Rocha CM, et al. (2018), que afirmam que há uma preocupação com a administração de medicamentos na urgência pediátrica e que a utilização de ferramentas tecnológicas ajuda a diminuir a ocorrência de erros na Unidade de Tratamento Intensivo Neonatal (UTIN) e em unidades de urgência e emergência.

Assim, o desenvolvimento de pesquisas voltadas para a urgência pediátrica, necessitam de incentivos e investimento na área tecnológica, tendo na criação de aplicativos móveis, a importância na otimização, melhora da qualidade do atendimento e redução de custos através de erros evitáveis, possibilitando também, acesso aos profissionais que não sejam pediátricos e atuem na urgência e emergência, contribuindo para a melhora na prescrição de cálculos de dose individualizados, dos quais não estejam familiarizados e que necessitam de agilidade dentro do atendimento.

Hoje em dia, o celular deixou de ser utilizado apenas para receber e enviar mensagens e / ou ligações e passou a ter novos usos. Ao expandir o escopo dos serviços, as pessoas podem navegar em redes sociais, acessar mapas, ler livros e compartilhar informações. A diversidade dessa possibilidade ajuda na atualização do cuidado e das práticas em saúde, permitindo formas mais seguras e eficientes no cotidiano assistencial (SILVA RH, et al., 2020; MARTINS CF, et al., 2017).

\section{CONCLUSÃO}

Este estudo desenvolveu e validou o aplicativo CALCULEPED para cálculo de dose pediátrica na unidade de terapia intensiva e urgência e emergência, sendo considerado pelos médicos pediatras, uma tecnologia de grande utilidade e aplicabilidade na prática clínica, auxiliando na redução da probabilidade de erros e otimizando o atendimento ao paciente pediátrico. Com o novo aplicativo visa-se diminuir a frequência da automedicação realizada por mães e responsáveis, assim como auxiliar na prática médica dos profissionais envolvidos. 


\section{REFERÊNCIAS}

1. BARBOSA AS. Mapeando as controvérsias que envolvem o processo de medicalização da infância. Psicol. soc. 2019.

2. BELO N, et al. Automedicação em idade pediátrica. Nascer e Crescer - Birth and Growth Medical Journal. 2017; 26(4): 234-9.

3. EMYINUMARU F, et al. Perfil e adequação do uso de antibacterianos em crianças internadas em hospital geral no sul do Brasil. Rev. paul. pediatr. 2019; 37(1): 27-33.

4. HEINZEL O, et al. Pediatric emergencies a major challenge. Med Klin Intensivmed Notfmed. 2015; 110(5): 326-327.

5. IPSM. Instituto para Práticas Seguras no Uso de Medicamentos potencialmente perigosos de uso hospitalar e ambulatorial - listas atualizadas [Internet]. 2015.

6. KAHN S, ABRAMSON EL. What is new in paediatric medication safety? Arch Dis Child. 2019 Jun; 104(6): 596-599.

7. MACEDO GPO, et al. Terapêutica medicamentosa para criança em serviço hospitalar de emergência. Acta Paul Enferm. 2015; 28(3): 237-42.

8. MANIERO HK, et al. Uso de medicamentos em crianças de zero a cinco anos de idade residentes no município de Tubarão, Santa Catarina. Rev Paul Pediatr. 2018;36(4):437-444.

9. MARTINS CF, et al. Utilização de medicamentos em crianças internadas em um hospital geral. Sci. med. 2017; 27(2).

10. MATOS JC, et al. Adesão às recomendações do uso de antibioticoprofilaxia e a ocorrência de infecção do sítio cirúrgico em pacientes pediátricos. Rev. méd. Minas Gerais. 2018; 28(9).

11. PEREIRA VSR, et al. Desenvolvimento, Utilização e Avaliação de uma Aplicação Móvel para Educação Médica: um Estudo de Caso em Anestesiologia. RENOTE. 2017; 15(1).

12. PIRES MCAC, et al. Linha do cuidado: a emergência pediátrica na perspectiva da integralidade do cuidado. Revista enfermagem atual. 2017; 80.

13. ROCHA CM, et al. Administração segura de medicamentos em neonatologia e pediatria: cuidados de enfermagem. Rev enferm UFPE. 2018; 12(12): 3239-46.

14. SERAFIM MPS, et al. Perfil das crianças usuárias do ambulatório de saúde mental do município de Içara - SC. Estud. Interdiscip. Psicol. 2019; 10(2): 192-209.

15. SIEBERT JN, et al. A mobile device app to reduce prehospital medication errors and time to drug preparation and delivery by emergency medical services during simulated pediatric cardiopulmonary resuscitation: study protocol of a multicenter, prospective, randomized controlled trial. Trials. 2019 Nov 20; 20(1): 634.

16. SILVA RH, et al. Aplicativos de saúde para dispositivos móveis: Uma revisão integrativa. Braz. J. Hea. Rev. 2020; v. 3(5): p. 11754-11765.

17. SOUZA ASC, et al. Eventos adversos e prática segura com medicação em pediatria: revisão integrativa. Revista enfermagem atual. 2018; 84.

18. VOLPATTO BM, et al. Erros de medicação em pediatria e estratégias de prevenção: revisão integrativa. Cogitare enferm. 2017; 22(1): 1-14.

19. WEGNER W, et al. Segurança do paciente no cuidado à criança hospitalizada: evidências para enfermagem pediátrica. Rev. Gaúcha Enferm. 2017; 38(1).

20. WILIAM W, et al. Segurança do paciente no cuidado à criança hospitalizada: evidências para enfermagem pediátrica. Rev Gaucha Enferm. 2017; 38(1). 